\title{
T4b Stage Finding
}

National Cancer Institute

\section{Source}

National Cancer Institute. T4b Stage Finding. NCI Thesaurus. Code C48734.

A general term that refers to a TNM finding of a primary tumor with direct invasion of adjacent structures. The definition of T4b TNM finding depends on the specific type of cancer that it refers to; for example, for bladder cancer it refers to a primary tumor that invades the pelvic wall and abdominal wall; for gastric cancer it refers to a primary tumor that invades adjacent structures; for colorectal cancer it refers to a primary tumor with direct invasion or adherence to other organs or structures. 